\title{
Intestinal Obstruction Due to Idiopathic Sclerosing Encapsulating Peritonitis: A Case Report
}

\author{
Ridvan Yavuz ${ }^{1}$; Sami Akbulut ${ }^{2, *}$; Mehmet Babur ${ }^{1}$; Firat Demircan ${ }^{1}$ \\ ${ }^{1}$ Department of Surgery, Diyarbakir Education and Research Hospital, Diyarbakir, Turkey \\ ${ }^{2}$ Department of Surgery, Liver Transplant Institute, Faculty of Medicine, Inonu University, Malatya, Turkey \\ ${ }^{*}$ Corresponding Author: Sami Akbulut, Department of Surgery, Liver Transplant Institute, Faculty of Medicine, Inonu University, Malatya, Turkey. Tel: +90-4223410660, Fax: +90-4223410036, \\ E-mail:akbulutsami@gmail.com
}

Received: July 11, 2014; Revised: March 23, 2015; Accepted: April 11, 2015

\begin{abstract}
Introduction: Sclerosing encapsulating peritonitis (SEP) is characterized by partial or complete encasement of small intestine by a thick fibrocollagenous membrane. Depending on underlying causes, SEP is divided into primary and secondary forms. Idiopathic SEP is also called idiopathic or abdominal cocoon syndrome. Herein we presented a case of idiopathic SEP.

Case Presentation:A90-year-old male patient presented to our emergency department with signs and symptoms of intestinal obstruction and dehydration. Physical examination findings, patient's age and plain abdominal radiography were consistent with tumoral obstruction or viscus perforation. Explorative laparotomy revealed a fibrous capsule encasing intestines as well as dense adhesions between intestinal loops. Since the overall condition of the patient was not well enough to allow a wide dissection and membrane excision, the operation was terminated after performing a limited loop ileostomy. Unfortunately, the patient was lost due to organ failure at the postoperative period. Conclusions: Despite advances in radiological techniques, the exact diagnosis in many cases is still made according to intraoperative findings and histopathological properties of the excised membrane. While some cases of SEP remain asymptomatic for years, most cases are characterized by recurrent bouts of acute, subacute or chronic intestinal obstruction. To our knowledge, the case presented here is the oldest patient with idiopathic SEP in the literature.
\end{abstract}

Keywords: Intestinal Obstruction; Encapsulating Peritoneal Sclerosis; Abdomen

\section{Introduction}

Sclerosing encapsulating peritonitis (SEP) is an entity characterized by encasement of small intestine by a fibrocollagenous membrane (1-5). It was first described as "peritonitis chronica fibrosa incapsulata" $(1,2)$. Depending on the underlying cause, the disorder is divided into primary (idiopathic) and secondary forms (1). The idiopathic form was named as abdominal cocoon syndrome (ACS) $(2,5)$. Affected patients most commonly present with acute, subacute or chronic attacks of intestinal obstruction $(1,3)$. The preoperative diagnosis is quite challenging and most cases are diagnosed intraoperatively (3). On the other hand, recent advances in radiological imaging modalities have now allowed preoperative diagnosis of SEP in some cases (4). A conservative treatment approach is the most suitable management strategy in asymptomatic idiopathic SEP. Various surgical options are used for symptomatic cases. In this paper, we aimed to present the oldest patient with idiopathic SEP in the literature.

\section{Case Presentation}

A 90-year-old Turkish male patient (Diyarbakir, Tur- key) presented to our emergency department in April 2011 with severe abdominal pain, nausea, vomiting and inability to defecate or pass gas. According to the information obtained from patient relatives, the patient had been admitted to separate healthcare facilities for a couple of times with abdominal pain and constipation for five years, but no pathology had been found by a variety of tests and examinations. The patient also lost at least 5 - $6 \mathrm{~kg}$ within the last year. His medical history was unremarkable except for hypertension controlled by a salt-free diet. His general appearance was cachectic, his abdomen distended and mucosae dry. Vital signs were as follows: blood pressure as $90 / 50 \mathrm{mmHg}$, pulse rate of $95 \mathrm{bpm}$ and body temperature of $37.9^{\circ} \mathrm{C}$. On physical examination, there was marked rebound tenderness and guarding in all quadrants. His laboratory tests were as follows; WBC 8.890 (neutrophil: 86.1\%), Hemoglobin $14 \mathrm{gr}$ | $\mathrm{dL}$, BUN $38 \mathrm{mg} / \mathrm{dL}$ and creatinine $0.8 \mathrm{mg} / \mathrm{dL}$. There were diffuse air-fluid levels in the abdomen on upright plain abdominal X-Ray (Figure 1, Table 1).

Thephysicalexaminationfindings, patient'sageandplain abdominal radiography were consistent with tumoral

Copyright (C) 2015, Iranian Red Crescent Medical Journal. This is an open-access article distributed under the terms of the Creative Commons Attribution-NonCommercial 4.0 International License (http://creativecommons.org/licenses/by-nc/4.0/) which permits copy and redistribute the material just in noncommercial usages, provided the original work is properly cited. 
Yavuz Ret al.

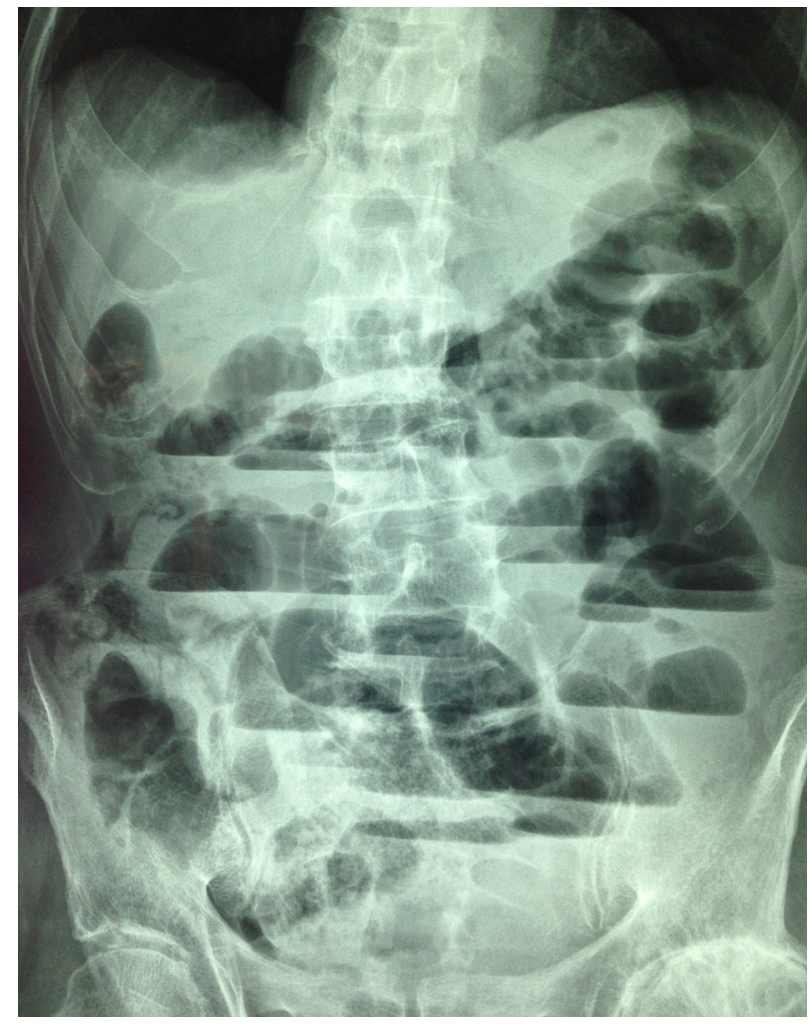

Figure 1. X-Ray Plain Abdominal Radiography Revealed Intestinal Obstruction With Small Bowel Air-Fluid Levels

obstruction or viscus perforation; therefore, patient was urgently taken into operation. A laparotomy was performed via midline incision, followed by abdominal exploration that revealed encapsulation of all intestinal segments by a dense, plate-like fibrous membrane that encased whole small intestine and caused dense adhesions between intestinal loops (Figure 2). Taken into consideration our previous experiences, we regarded this appearance as abdominal cocoon syndrome. As the fibrocollagenous membrane was densely adhered to both intestinal surface and interloop distance, excision and adhesiolysis could only be applied to a limited region. Considering the overall medical condition of the patient, we did not perform membrane excision and adhesiolysis that would prolong the operation time. A loop ileostomy was established and the operation was terminated. The patient was admitted to intensive care unit owing to his poor general status. Despite aggressive fluid and electrolyte replacement attempts and administration of wide-spectrum antibiotics, the patient died as a consequence of septic

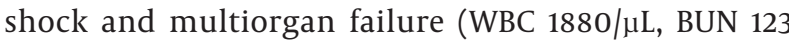
$\mathrm{mg} / \mathrm{dL}$ creatinine $2.92 \mathrm{mg} / \mathrm{dL}$ and albumin $2.4 \mathrm{gr} / \mathrm{dL}$ ) three days after the operation.
Table 1. Patients' Characteristics

\begin{tabular}{lc}
\hline Variables & Results \\
\hline Age, $\mathbf{y}$ & 90 \\
Gender & Male \\
\hline Pulse rate, bpm & 95 \\
\hline Blood pressure, $\mathbf{m m H g}$ & $90 / 50$ \\
\hline Body temperature, C & 37.9 \\
\hline WBC, $/ \boldsymbol{L} \mathbf{L}$ & 8890 \\
\hline Hb, gr/dL & 14 \\
\hline BUN, $\mathbf{m g} / \mathbf{d L}$ & 38 \\
\hline Creatinine, $\mathbf{m g} / \mathbf{d L}$ & 0.8 \\
\hline X-Ray radiography & Air-fluid levels \\
\hline Physical Examination & Acute abdomen \\
\hline
\end{tabular}

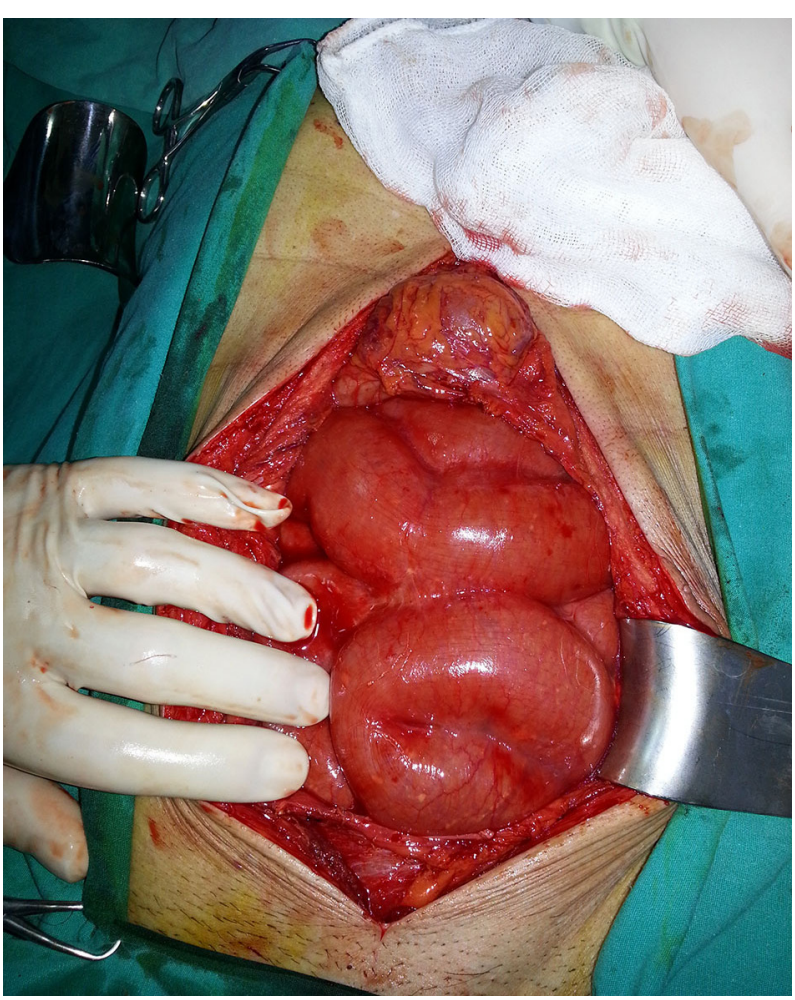

Figure 2. Intraoperative Photography Showing Encapsulated Small Bowe (Cocoon Like Dense Fibrous Membrane)

\section{Discussion}

Depending on the underlying causes, SEP is divided into primary (idiopathic) and secondary forms (2, 6-9). The idiopathic form of the disease was named as 'abdominal cocoon syndrome' in $1978(2,3,8,10)$. Idiopathic SEP is mostly observed in female children of adolescent age living in tropical and subtropical countries, although may be seen in children living in temperate regions or adults of advanced age (1). In a study that presented the youngest 
Yavuz R et al.

case series to date, the disease was 7 times more common in men compared to women (3). Although many theories have been put forward to explain the etiology of the disease, the exact etiology has remained to be proven. In women, retrograde menstruation, retrograde extension of fallopian infections or viral gynecological infections have been implicated as probable causes of disease $(2,3)$. In contrast, the exact mechanism has remained unclear in men. The most common known cause of secondary SEP worldwide is peritoneal dialysis (2). The main objective of numerous articles on secondary SEP in the literature is the association between peritoneal dialysis and SEP. Abdominal tuberculosis is responsible for a sizeable part of secondary SEP in underdeveloped countries (9). Previous surgeries, sarcoidosis, gastrointestinal tumors, SLE, FMF, fibrogenic foreign body, beta-blocker use, ventriculoperitoneal and peritoneovenous shunts, orthotopic liver transplantation and recurrent peritonitis are among other rare causes $(5-7,9)$. However, the patient presented here had no apparent cause, neither in medical history nor in the microbiological tests. Thus, we considered the case as idiopathic SEP.

While some cases of SEP have an asymptomatic course, most exhibit symptoms characterized by recurrent acute, subacute or chronic attacks of gastrointestinal obstruction $(1,2,7)$. Among the symptoms of the disease, abdominal pain, nausea, vomiting, loss of appetite, weight loss, distention and inability to defecate or pass gas are the primary symptoms $(1,2)$. Occasionally, a soft and painless mass lesion can be palpated in some patients $(2,6)$. To date, two cases of intestinal perforation secondary to SEP have been reported, one being idiopathic and the other secondary to tuberculosis (1).

The first and most important steps towards diagnosis of SEP are awareness and clinical suspicion proportional to experience and knowledge of the disease $(1,7)$. Among patients presenting to emergency department with intestinal obstruction, 60 - $80 \%$ are caused by postoperative adhesions, while only $6 \%$ are caused by unusual causes (2). Idiopathic SEP forms only a small part of unusual cases leading to intestinal obstruction (2). Therefore, clinical suspicion is the most important issue in diagnosing SEP. Apart from clinical history and suspicion, the most frequently diagnostic tools are X-Ray films, barium passage radiographies, ultrasonography (USG) and computed tomography $(\mathrm{CT})(1,2)$. As the case presented here, cases with signs and symptoms of intestinal obstruction have small bowel dilatation and air-fluid levels on abdominal X-Ray films. Air-fluid levels in elderly patients who have not been operated previously suggest tumor obstruction or chronic mesenteric ischemia (1). An accordion pattern and cauliflower appearance on barium passage radiographies of small intestine may give a clue of SEP $(1,2,7)$. However, barium radiographies may not always be possible in cases with full obstruction. Abdominal USG may demonstrate dilated small bowel segments surrounded by a thick, hypoechogenic fibrous membrane $(2,7)$. CT is currently the most useful radiological method for diagnosis of SEP(2). Especially multidetector CT with excellent image quality on coronal, sagittal and axial planes may provide the best results. The characteristic CT sign is the appearance of loops of small intestine that conglomerate at midline and are encased by a dense mantle without peripheral contrast uptake (2). The major obstacles before CT include presentation of many patients to emergency departments with signs and symptoms of intestinal obstruction, physical examination findings consistent with acute abdomen and lack of CT in many centers. Considering all these factors, it can be concluded that the preoperative diagnosis is still difficult in symptomatic cases with SEP and most patients are diagnosed by intraoperative signs and histopathological findings (5).

Prudent follow-up is all that is needed in asymptomatic cases with idiopathic SEP (7). Conservative management is the most suitable therapy in mildly symptomatic cases. Such cases are best managed by intestinal rest, nasogastric decompression and nutritional support (enteral or parenteral) $(3,11)$. Cases with no regression of symptoms may be treated with antiinflammatory or antifibrogenic drugs such as tamoxifen, steroids, colchicine, azathioprine and mycophenolate mofetil (11). However, these treatment protocols have mainly been used in secondary SEP and there are no sufficient data to justify their use in idiopathic SEP. To my opinion and experience, antifibrogenic medications can be administered in Types II and III ACS. Surgical therapy is used in markedly symptomatic cases or cases with SEP diagnosis confirmed during laparotomy is still debated. Different options such as membrane excision + adhesiolysis, resection + anastomosis, resection + anastomosis + protective enterostomy can be used alone or in combination, depending on the condition of patient. Intestinal resection should be avoided owing to certain complications including anastomosis leaks and short bowel syndrome since resection is usually not essential and unnecessary resections increase morbidity and mortality $(3,7)$. The role of laparoscopy in the management of SEP is unclear. In the literature, there are a limited number of case reports in which the authors reportedly performed successful membrane excision and adhesiolysis. One additional advantage of laparoscopy is that it can be used for diagnostic purposes in cases with unclear diagnosis (8).

In conclusion, ACS forms a minority of unusual conditions that lead to intestinal obstruction. Preoperative diagnosis is a true challenge and most reported cases have been incidentally diagnosed during laparotomy. Fortunately, clinicians possess some diagnostic radiological methods that aid in diagnostic process. While a conservative approach is the best in mildly symptomatic cases, symptomatic cases frequently require surgical management. A minimally invasive approach should be the norm to avert troublesome complications such as anastomosis leaks and short bowel syndrome. 


\section{Authors' Contributions}

Study concept and design: Sami Akbulut and Ridvan Yavuz. Analysis and interpretation: Sami Akbulut and Firat Demircan. Drafting of the manuscript: Sami Akbulut, Ridvan Yavuz and Mehmet Babur. Critical revision of the manuscript for important intellectual contest: Sami Akbulut and Firat Demircan.

\section{Funding/Support}

This study was not supported financially and did not receive any kind of grants.

\section{Financial Disclosure}

The authors declared no financial interests related to the material in the manuscript.

\section{References}

1. Akbulut S, Yagmur Y, Babur M. Coexistence of abdominal cocoon, intestinal perforation and incarcerated Meckel's diverticulum in an inguinal hernia: A troublesome condition. World J Gastrointest Surg. 2014;6(3):51-4.

2. Tannoury JN, Abboud BN. Idiopathic sclerosing encapsulat- ing peritonitis: abdominal cocoon. World $J$ Gastroenterol. 2012;18(17):1999-2004.

3. Li N, Zhu W, Li Y, Gong J, Gu L, Li M, et al. Surgical treatment and perioperative management of idiopathic abdominal cocoon: single-center review of 65 cases. World J Surg. 2014;38(7):1860-7.

4. Solak A, Solak I. Abdominal cocoon syndrome: preoperative diagnostic criteria, good clinical outcome with medical treatment and review of the literature. Turk J Gastroenterol. 2012;23(6):776-9.

5. Serter A, Kocakoc E, Cipe G. Supposed to be rare cause of intestinal obstruction; abdominal cocoon: report of two cases. Clin Imaging. 2013;37(3):586-9.

6. Akbulut S. Accurate definition and management of idiopathic sclerosing encapsulating peritonitis. World J Gastroenterol. 2015;21(2):675-87.

7. Xu P, Chen LH, Li YM. Idiopathic sclerosing encapsulating peritonitis (or abdominal cocoon): a report of 5 cases. World J Gastroenterol. 2007;13(26):3649-51.

8. Ertem M, Ozben V, Gok H, Aksu E. An unusual case in surgical emergency: Abdominal cocoon and its laparoscopic management. J Minim Access Surg. 2011;7(3):184-6.

9. Singh B, Gupta S. Abdominal cocoon: a case series. Int J Surg. 2013;11(4):325-8.

10. Wei B, Wei HB, Guo WP, Zheng ZH, Huang Y, Hu BG, et al. Diagnosis and treatment of abdominal cocoon: a report of 24 cases. Am J Surg. 2009;198(3):348-53.

11. Habib SM, Betjes MG, Fieren MW, Boeschoten EW, Abrahams AC, Boer WH, et al. Management of encapsulating peritoneal sclerosis: a guideline on optimal and uniform treatment. Neth J Med. 2011;69(11):500-7. 